\title{
Formal Models for Real People
}

\author{
Michiel van Lambalgen • Marian Counihan
}

Published online: 1 July 2008

(C) The Author(s) 2008

Logic may have played an important role in the genesis of cognitive science, but its importance for this field has progressively diminished, as a consequence of many different pressures. Some of the arguments, in arbitrary order, are as follows:

(1) Experiments with reasoning tasks, such as the famous Wason selection task (Wason 1968), show that logical form is not a determinant of reasoning

(2) Logic cannot deal with vagueness and prototypicality

(3) Logic cannot deal with uncertainty and must be replaced by probability theory, which is after all the calculus of uncertainty

(4) What we know about the neocortex suggests that the computations executed by the brain are very different from logical computations

(5) The computational complexity of logic is too high for logic to be a realistic model of cognition

Logicians reading this will have already shaken their head at some of these claims; the aim of this special issue is to provide empirical and formal counterevidence to them, and others in a similar vein. In this introduction, we examine in greater detail the argument 1, using it as a springboard to defend a restored central role for logic, and more generally formal models of cognition (in a sense which we make exact later on), in cognitive science.

We begin by discussing the selection task and its aftermath. Wason's original task was presented by means of a form as depicted in Fig. 1. In order to appreciate the tremendous difficulty posed by this task, the reader-who has probably seen the task before-should realise that this is all the information provided to the subjects. This experiment has been replicated many times.

M. van Lambalgen $(\varangle) \cdot$ M. Counihan

ILLC / Department of Philosophy, University of Amsterdam, Nieuwe Doelenstraat 15,

1012 CP Amsterdam, The Netherlands

e-mail: M.vanLambalgen@uva.nl 
Fig. 1 Wason's selection task

Cards:

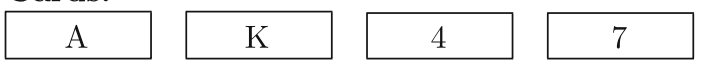

Below is depicted a set of four cards, of which you can see only the exposed face but not the hidden back. On each card, there is a number on one of its sides and a letter on the other.

Also below there is a rule which applies only to the four cards. Your task is to decide which if any of these four cards you must turn in order to decide if the rule is true. Don't turn unnecessary cards. Tick the cards you want to turn.

Rule: If there is a vowel on one side, then there is an even number on the other side.

If one formulates the rule If there is a vowel on one side, then there is an even number on the other side. as an implication $p \rightarrow q$, then the observed pattern of results is typically given as in Table 1 . Wason believed there to be only one 'logically correct' answer, namely $p, \neg q$, and concluded that according to this standard, the vast majority of reasoners are irrational. In addition, the selection task was used to argue that most adults do not reach what Piaget (1953) considered the pinnacle of cognitive development, the formal operational stage, which is basically mastery of classical propositional logic. Not only do subjects typically fail to master the modus tollens inference supposed to be at work here, they do not even have a workable concept of logical form to guide their reasoning. For if Wason's 'abstract' rule If there is a vowel on one side, then there is an even number on the other side. is replaced by the 'concrete' rule if you want to drink alcohol, you have to be over 18, performance suddenly jumps to more acceptable levels, around 75\%. These two reasoning tasks-'abstract' and 'concrete' - are considered to have the same logical form. So, the argument goes, if logical form determines performance in a task, performance on these tasks must be comparable. The data show that performance differs wildly, whence logical form is irrelevant to reasoning. A tacit inference followed: if logic plays no role in reasoning, why would it play a role in other domains of cognition?

We must first note that so far we have been talking about logic in the singular, as is common in cognitive science, which proceeds mostly as if classical logic were the only game in town. But to appreciate logic's place in cognitive science, a broader conception of logic is necessary. This involves acknowledging the need for many different logics, varying in syntax, semantics and definition of validity (Stenning and van Lambalgen 2008). In fact, the impression that logic is useless in cognitive science is for the most part due to the uncritical identification of logic with classical logic, or in any case with a system of rules. In logic itself this conception was finally laid to rest with the advent of 'model theoretic logics', for which see (Barwise 1985).

Table 1 Typical scores in the selection task

\begin{tabular}{lllll}
\hline$p$ & $p, q$ & $p, \neg q$ & $p, q, \neg q$ & misc. \\
\hline $35 \%$ & $45 \%$ & $5 \%$ & $7 \%$ & $8 \%$ \\
\hline
\end{tabular}


This need for many logics arises because at a suitably abstract level cognitive functions, i.e. information processing tasks, can be usefully described in $a$ logic, not necessarily classical, and not necessarily given by a system of rules. To see why this can be so, consider David Marr's three levels of cognitive inquiry Marr (1982, Chap. 1):

(1) identification of the information processing task as an input-output function; ${ }^{1}$ this competence model expresses what the information processing task considered is supposed to do

(2) specification of an algorithm which computes that function

(3) neural implementation of the algorithm specified ${ }^{2}$

As an example of how a competence model can be specified, consider (a particular view of) language comprehension. Here, the input consists of natural language discourse, and the output consists in discourse models, or more technically, 'discourse representation structures' (DRS) (Kamp and Reyle 1993; Hamm et al. 2006). Both input and output can be specified in terms of a formal language. The formal structure of the input language is simple: concatenation of sentences. For the output we need a language describing the various ways in which DRSs can be combined. The specific 'competence' aspect is given by the norm relating input and output: if the input discourse is true in a model $\mathcal{A}$, the output DRS can be embedded in $\mathcal{A}$. As regards the second level, there are algorithms transforming natural language discourse into a corresponding DRS — corresponding in the sense that the competence model is satisfied. A neural implementation does not exist yet-but see below.

To return to the competence level, observe that the norm relating input and output can be viewed as a definition of validity of 'arguments' $S_{1}, \ldots, S_{n} \vdash K$, where the $S_{i}$ are sentences and $K$ is (a sentence describing) a DRS. Thus we have all the ingredients of a logic here: syntax, semantics, validity. But why is this a useful way of looking at the competence level?

One useful feature of such a fully specified competence model is that it allows a correctness proof for the algorithm of the second level. Equally importantly, in setting up the competence model formally one may uncover assumptions which restrict its scope unduly. An example is in order here. The formal model for discourse comprehension sketched earlier has as a consequence that, as the discourse grows, the set of discourse models forms a monotonic chain. Assume that the given discourse grows from $S_{1}, \ldots, S_{n}$ to $S_{1}, \ldots, S_{n}, S_{n+1}$, and that the semantic force of the comma is conjunction, so that ' $S_{1}, \ldots, S_{n}, S_{n+1}$ is true on $\mathcal{A}$ ' implies ' $S_{1}, \ldots, S_{n}$ is true on $\mathcal{A}$. Let $K_{n}$ be the discourse model corresponding to $S_{1}, \ldots, S_{n}$, and $K_{n+1}$ that for $S_{1}, \ldots, S_{n}, S_{n+1}$. Then both $K_{n}$ and $K_{n+1}$ are embeddable in $\mathcal{A}$, and since the universe of $K_{n}$ is a subset of that of $K_{n+1}$, it follows that $K_{n}$ must be a substructure of $K_{n+1}$. Thus the sequence of discourse models is monotonic.

As soon as one realises this, one sees that the proposed competence model lacks cognitive plausibility. Consider the simple discourse:

\footnotetext{
1 Marr calls this the 'computational level', but this term is infelicitous in view of what the next level does. We prefer the term 'competence model' instead.

2 This tripartite scheme of explanation should be read neither bottom-up nor top-down, but as a set of constraints; for example, the algorithm may be determined both by the competence model and by what is known about neural computation.
} 
Some real estate agents are crooks. In fact all of them are.

Gricean maxims tell you that after the first sentence the discourse model verifies 'Some real estate agents are not crooks', a statement that is flatly denied by the second sentence. So here the progression of discourse models cannot be monotonic. While this example is from pragmatics, the following is squarely inside semantics, and concerns the meaning of the English progressive (Baggio and van Lambalgen 2007).

The door of the living-room was closed. Inside the radio played classical music.

The girl was writing letters [*] when her friend spilled coffee on the tablecloth/paper.

Behavioural data show that if the sentence ends with 'tablecloth', $75 \%$ of subjects conclude that in the end a letter was written, whereas if the final word is 'paper', this percentage drops to $37 \%$. The most parsimonious explanation for this is that in the discourse model of the discourse up to the point [*], a finished letter is introduced, so that after seeing 'paper', but not after seeing 'tablecloth', that entity must be removed again. ${ }^{3}$ Therefore this sequence of discourse models is non-monotonic. It is argued in Baggio and van Lambalgen (2007), and that a fairly well-established discourse processing principle, the 'principle of immediacy', which says that

every source of information that constrains the interpretation of an utterance (syntax, prosody, word-level semantics, prior discourse, world knowledge, knowledge about the speaker, gestures, etc.) can in principle do so immediately [i.e. before sentence boundary], (Hagoort and van Berkum 2007)

forces a non-monotonic progression of discourse models. Again, this shows that the earlier competence model, in particular its definition of validity, is not cognitively plausible.

In the face of such difficulties, a common tactic in cognitive science is to drop all reference to a competence model and be content with an algorithm instead. The downside of this tactic is that one ends up with a bunch of specialised algorithms, and that commonalities between tasks may disappear from view. So what has to replace the discarded competence model? The definition of validity cannot be direct comparison with the real world, but it may come from internal considerations on what is the 'best' notion of non-monotonicity from a cognitive point of view.

More generally, this example serves to illustrate that cognitively realistic formal models are possible, and moreover, that they are needed. They provide the requisite level of information-theoretic description, thereby making modelling assumptions explicit, and eventually enabling commonalities across cognitive tasks and domains to be investigated and described. After all, the goal of cognitive science is a unified theory of cognition. We hope that the articles in this special issue of the journal provide suitable formal tools to move further towards this goal.

Open Access This article is distributed under the terms of the Creative Commons Attribution Noncommercial License which permits any noncommercial use, distribution, and reproduction in any medium, provided the original author(s) and source are credited.

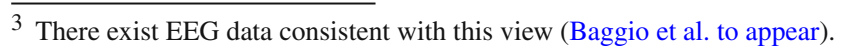




\section{References}

Baggio, G., \& van Lambalgen, M. (2007). Processing consequences of the imperfective paradox. Journal of Semantics, 24(3). doi:10.1093/jos/ffm005.

Baggio, G., van Lambalgen, M., \& Hagoort, P. Computing and re-computing discourse models: an ERP study. Journal of Memory and Language (to appear)

Barwise, J. (1985). Model-theoretic logics: Background and aims. In J. Barwise \& S. Feferman (eds.), Model-theoretic logics (Chap. I). New York: Springer-Verlag.

Hagoort, P., \& van Berkum, J. (2007). Beyond the sentence given. Philosophical Transactions of the Royal Society of London. Series B: Biological Sciences, 362(1481), 801-811 (2007). doi:10.1098/rstb.2007. 2089.

Hamm, F., Kamp, H., \& van Lambalgen, M. (2006). There is no opposition between formal and cognitive semantics. Theoretical Linguistics, 32(1):1-40. Pages 41-115 have comments on this target article.

Kamp, H., \& Reyle, U.(1993). From discourse to logic, introduction to modeltheoretic semantics of natural language, formal logic and discourse representation theory, Part 1. Studies in linguistics and philosophy(Vol. 42). Dordrecht: Kluwer Academic Publishers.

Marr, D. (1982). Vision: A Computational investigation into the human representation and processing of visual information. San Fransisco: W.H. Freeman.

Piaget, J. (1953). Logic and psychology. Manchester: Manchester University Press.

Stenning, K., \& van Lambalgen, M. (2008). Human reasoning and cognitive science. Cambridge, MA: MIT Press.

Wason, P. C. (1968). Reasoning about a rule. Quarterly Journal of Experimental Psychology, 20, $273-281$. 